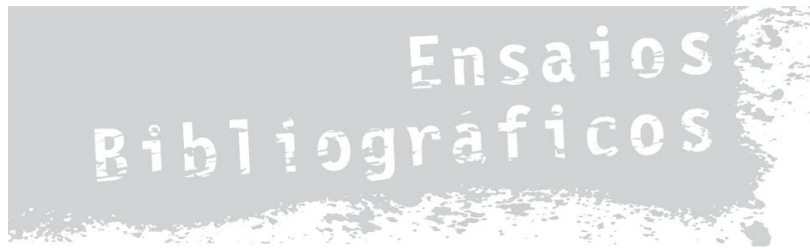

\title{
As percepções sobre psicofármacos na Índia: pensando Eating Drugs, de Ecks
}

\author{
Marcos Freire de \\ Andrade Neves (UFRGS)
}

\begin{abstract}
"Qual o problema de tomar remédio?", ele perguntou. As pílulas são apenas "suplementos nutricionais" para o cérebro. Assim como qualquer pessoa deve tomar vitaminas quando comida normal não foi suficiente, qualquer pessoa deve tomar suplementos de comida para o cérebro quando a nutrição foi deficiente. Os remédios receitados foram moner khabar, e opor-se a eles foi tão imprudente quanto opor-se a tomar vitamina $\mathrm{C}$ enquanto gripado' 1. (Ecks 2014: 178-181, tradução minha²).
\end{abstract}

Psicofármacos são alimentos para a mente (moner khabar, em bengali), dizem os médicos a seus pacientes na cidade de Calcutá, Índia. Tal premissa é o ponto de partida de Stefan Ecks, cujo livro Eating Drugs: Psychopharmaceutical Pluralism in India (Biopolitics: Medicine, Technoscience, and Health in the 21st Century) tem por objetivo explorar os modos pelos quais os usos de psicofármacos são percebidos na interação entre médicos e pacientes. Ecks, diretor do Programa de Antropologia Médica na Universidade de Edimburgo, Escócia, empreende uma etnografia na cidade de Calcutá - antiga capital da Índia britânica e atualmente habitada por cerca de 4.500 .000 pessoas -, durante a qual entrevistou profissionais e usuários de diferentes sistemas de medicina.

Conquanto na Índia sejam reconhecidos sete sistemas nacionais de medicina, Ecks circula, basicamente, por entre três destes: Ayurveda, homeopatia e alopatia. A premissa exposta por Ecks - e reforçada pelos inúmeros depoimentos que sustentam a sua argumentação -, é percebida no interior de uma intrincada interação entre fundamentos medicinais e econômicos, modos de atendimento, diagnóstico e tratamento compartilhados e disputados pelos diferentes sistemas de medicina. Premissa que sugere uma simplificação para estas interações (equiparar medicamentos com comida enquanto metáfora que possibilita maior proximidade entre os pacientes e os médicos), mas que, no entanto, é metonímica de complexas compreensões médicas, religiosas e tradicionais indianas sobre o funcionamento do corpo e da mente. 
“Aos pacientes, Dr. Roy disse que o cérebro precisava comida suficiente para pensar apropriadamente, assim como o estômago precisava comida para manter o corpo funcionando" (Ecks 2014: 145-148, tradução minha). Através deste e outros depoimentos, Ecks questiona se os médicos devem insistir em explicações baseadas em um purismo científico ou devem procurar histórias persuasivas capazes de auxiliar o entendimento de seus pacientes. Contudo, não se trata apenas de questionar modos pelos quais o entendimento pode ser alcançado, mas sim, sobretudo, de ponderar sobre a própria premência do entendimento. Até que ponto é o entendimento - por parte dos pacientes - acerca dos diagnósticos e tratamentos algo necessário ou desejável?

Torna-se imprescindível, portanto, compreender os pontos de aproximação e discordância entre médicos e pacientes: "Como ideias mudam através da persuasão, dissimulação ou engano; e como novas metáforas, como 'comida para a mente', são tentativas de superar essas divisões", escreve o autor, "'Comida para a mente' é uma forma de linguagem psiquiátrica que se apropria do discurso popular para superar resistências" (Ecks 2014: 223-225). A ideia de metáfora desempenha um papel fundamental no trabalho de Ecks, pois representa a possibilidade de união entre uma percepção cotidiana - e tradicional - e uma linguagem especializada. Associar as duas linguagens por meio da ideia de comida une, por um lado, o saber popular acerca da centralidade do sistema digestivo para a manutenção da boa saúde e, pelo outro, a diminuição da resistência comum contra o uso de psicofármacos. Se, conforme argumenta Ricoeur, a metáfora consiste na apresentação de "uma ideia sob o signo de outra ideia mais impressionante ou mais conhecida" (Ricoeur 1983: 94), a associação em torno da ideia de "comida" desempenharia papel semelhante.

A dificuldade em pensar através da ideia de metáfora, tal como projetou o autor, decorre da necessidade de estabelecer um eixo principal - um domínio fonte - que seria, posteriormente, apropriado por meio de outros termos. Haveria uma relação entre significados principal e secundários. Poder-se-ia, portanto, pensar na elaboração da ideia "comida para a mente" nos termos de uma estratégia desenhada a partir da compreensão de um jogo de interessamentos. Isto é, uma espécie de tradução (Domènech; Tirado 1998) - realizada por médicos que tem por objetivo convencer pacientes ao uso de medicamentos aos quais, de outro modo, manifestariam resistência. Uma tradução a partir do interesse médico em ampliar seu público é desenhada com base na compreensão de interesses populares (relativos à importância da ideia de comida e do sistema digestivo para a boa saúde). 0 resultado justaposto de lógicas múltiplas (Law et. al. 2013).

Há gerações, o sistema digestivo ocupa posição central na concepção de boa saúde na Índia. Preserválo de modo saudável tem efeitos coextensivos ao corpo como um todo, e nessa dinâmica a alimentação é decisiva. Não à toa, até a década de 1980, remédios ayurvédicos (derivado de Ayurveda, sistema de medicina indiano milenar cujo nome, em sânscrito, significa "ciência da vida") eram produzidos com foco em problemas sexuais e digestivos (dois aspectos intimamente relacionados na concepção da Ayurveda). Os medicamentos - equiparados à comida - fornecem uma importante chave analítica e metodológica para a compreensão dos diferentes significados e usos a eles atribuídos.

Com o intuito de adentrar esse intrincado complexo de relações que tece as percepções e usos cotidianos de medicamentos - de modo geral - e psicofármacos - de modo específico -, Ecks propõe um caminho 
metodológico similar à biografia social das coisas, nos termos de Appadurai (Ecks 2014: 242). Trata-se de buscar um ponto de referência estável a partir do qual traçar uma trajetória de análise. Se, conforme argumenta Hacking, as classificações médicas interagem com o que estão classificando, torna-se demasiadamente difícil saber o que veio primeiro: “o rótulo da doença, as pessoas sendo rotuladas como portadoras da doença, as instituições desenhadas para lidar com essa doença, o corpo de conhecimento que sustenta o rótulo da doença ou seus especialistas" (Ecks 2014: 236-238). Nesse sentido, os remédios oferecem à análise maior estabilidade referencial do que as próprias doenças (Ecks 2014: 246-247).

Embora não integre, ao menos diretamente, o corpo teórico de Eating Drugs, identificar-se no medicamento um ponto de estabilidade frente à instabilidade das doenças com base no argumento de Hacking sugere uma aproximação com o trabalho de Mol. Para a autora, não há uma doença - ou, ampliando o escopo, uma realidade - que anteceda as suas práticas. Conquanto Ecks tenha lançado mão da etnografia a partir de um ponto de referência estável, é justamente na instabilidade das doenças que Mol foca sua empresa etnográfica. O desafio da autora é pensar como superar o perspectivismo (há uma doença e diferentes perspectivas que a circundam) e alcançar etnograficamente a doença em si $(2008 ; 2002)$.

Com isso em vista, parece haver aqui dois modos de operacionalizar etnograficamente a doença: a estratégia de Mol, focada nas praticalidades, materialidades e eventos que a performa e a de Ecks, para quem o foco nos remédios oferece uma espécie de engenharia reversa na cadeia de atendimento médico. A partir do remédio escolhido, deve-se questionar quem o fabrica e distribui, quem o comercializa, quem o prescreve e quem o utiliza (Ecks 2014: 248-252). A partir dessas relações, a doença surge como pano de fundo.

Para além da estratégia metodológica escolhida, Eating Drugs apresenta um interessante quadro dos sistemas medicinais indianos, suas proximidades e distanciamentos. A despeito das diferentes trajetórias históricas e fundamentos calcados na tradição ou na ciência, os três sistemas ao redor dos quais o trabalho foi circunscrito conversam entre si, compartilham pacientes e meios de diagnóstico - o que não significa insinuar uma ausência de conflitos entre seus agentes. É justamente em decorrência dessa possibilidade de diálogo entre os agentes de diferentes sistemas de medicina que o argumento central de Ecks toma forma:

(...) a plausibilidade de chamar psicofármacos "comida para a mente" depende menos das ideias dos pacientes sobre psicofármacos biomédicos como balas mágicas do que em profundas camadas de combinação entre noções biomédicas, ayurvédicas, homeopáticas e populares. (Ecks 2014: 258-260)

E é com o intuito de apresentar tais camadas de combinação que o autor estrutura o seu livro. No primeiro capítulo, "Popular Practice: The Belly and the 'Bad Mind'" ("Prática Popular: A barriga e a 'mente ruim'"), Ecks apresenta um princípio bengali que será fundamental no decorrer de sua argumentação: "a barriga é o centro somático da boa saúde" (Ecks 2014: 446). A noção popular referente à interação entre corpo e mente acaba por atuar como um princípio norteador no momento de elaborar diagnósticos, direcionando perguntas a serem feitas pelos médicos - em maior ou menor grau dependendo do sistema no qual estão inscritos - e influenciando no uso dos medicamentos feito pelos pacientes. 
Ummon [mente] controlado e em pazé visto como uma precondição à vida saudável. Quando você não está preocupado com nada, quando você mantém a sua mente concentrada, você será sempre saudável. (...) "Se a mente é ruim, o corpo é doente". (...) Por sua vez, poder mental (moner jor) pode superar doenças. Quando o calor do estômago está sob controle, a mente pode permanecer sob controle também. De forma mais simples, isso é expresso no dito bengali bhudi thanda thakle, mudi thanda thake ("Se a barriga permanece fria, a cabeça permanece fria"). (Ecks 2014: 464).

É com base nessa prática popular que a utilização da ideia de comida enquanto metáfora aos psicofármacos toma sua inspiração. Uma ideia pré-científica, derivada de saberes tradicionais e valores culturais que acabam por orientar sua apropriação por uma fração do corpo médico em termos de uma história persuasiva. Persuasão que busca estabelecer um vínculo entre médico e paciente acerca do diagnóstico elaborado e do medicamento indicado sem, com isso, adentrar os pormenores. Detalhes são evitados:

Ele [Dr. Sengupta, médico da Ayurveda] preferia não confrontar seus pacientes com um diagnóstico psicológico: 'Se eu os disser, eles pensariam: 'Oh, esse médico não pode diagnosticar apropriadamente'. Ao invés disso, ele diagnostica um problema físico e prescreve um placebo. (Ecks 2014: 1412-1413).

Segundo o conhecimento tradicional, frisa o autor, mente e corpo são entidades diferentes que, no entanto, interagem. Uma interação benéfica quando a mente exerce controle sobre o corpo e maléfica quando este exerce controle sobre a mente (Ecks 2014: 515). A descrição deste conhecimento tem continuidade ao longo do segundo capítulo, "Ayurveda: 'You Are the Medicine'" ("Ayurveda: 'Você é o remédio'"), dedicado ao sistema médico tradicional indiano. Ecks nos apresenta a história de Dr. Sengupta, um kaviraj (médico da Ayurveda), que desde a infância sofria de moderada dor em seu estômago. Sempre pensada em termos de um simples problema gástrico, a dor aumentou após os seus sessenta anos, o que o levou a procurar outra opinião ayurvédica. Acidez, foi o diagnóstico dado pelo kaviraj. Insatisfeito, procurou uma terceira opinião: alargamento do fígado, foi o novo diagnóstico. Ainda descontente com os diagnósticos recebidos, Dr. Sangupta procurou um alopata e se submeteu a uma série de exames - raio-x, endoscopia, biópsia, etc. - que acabaram por indicar um câncer em seu esôfago.

A história relatada por Ecks tem uma importância central: indica a complementaridade dos sistemas de medicina. Embora Dr. Sengupta fosse kaviraj, não hesitou em procurar outro sistema de medicina após concluir que a Ayurveda não mais poderia ajudá-lo: "Se eu não posso fazer, eu os mando para a alopatia. Você não pode ignorar a alopatia, é tão importante, é compulsória. Especialmente em casos de emergência" (Ecks 2014: 1402). Isso indica uma espécie de caminho relativo aos sistemas de medicina, o qual envolve consultas complementares entre Ayurveda, homeopatia e alopatia. A escolha pelo caminho a ser trilhado envolve, geralmente, questões econômicas e de temporalidade: a urgência em ter o problema de saúde resolvido (nesse quesito, alopatia é considerada a forma mais rápida - embora traga consigo efeitos colaterais; enquanto homeopatia é considerada lenta, porém isenta de efeitos adversos) e o preço das consultas e dos tratamentos.

A complementaridade dos sistemas pode ser ilustrada, novamente, pelo próprio Dr. Sengupta, que passou a trabalhar em uma empresa farmacêutica que descobriu na produção de uma linha de produtos ayurvédicos uma maior fonte de lucratividade quando comparada à produção de genéricos biomedicinais. Uma mesma 
empresa fabricando remédios para sistemas médicos distintos, em um processo de industrialização de uma prática artesanal com receitas antes transmitidas familiarmente (Ecks 2014: 1384).

No terceiro capítulo, "Homeopathy, Immaterial Medicines" ("Homeopatia, Medicinas Imateriais"), é oferecido um quadro acerca do segundo sistema de medicina mais popular na Índia, atrás apenas da alopatia (Ecks 2014: 2020). Com origem na Alemanha, a homeopatia foi desenvolvida pelo médico Samuel Hahnemann e difundida na Índia desde o começo do século XIX (o grau de difusão pode ser ilustrado pelo comentário de Dr. Bannerjee, transcrito por Ecks: "Nesse país, se você jogar uma pedra, ela atingirá um homeopata" [Ecks 2014: 2026]).

Diferentemente da alopatia (que, argumenta o autor, teve seu nome cunhado por Samuel Hahnemann para designar "medicina antiga" [Ecks 2014: 2054]), a homeopatia não possui efeitos colaterais. Seus princípios descritos no Organon, texto fundador do sistema e publicado no início do século XIX - são contrários à utilização excessiva (isto é, em altas doses) e combinada de medicamentos, que devem, primordialmente, atingir as causas do problema de saúde e não os seus sintomas. Parece haver, entre estes dois sistemas de medicina, diferentes concepções de corpo. Enquanto a alopatia age na dependência de um diagnóstico específico que leva à prescrição de um medicamento correspondente, agindo apenas com relação ao problema identificado, a homeopatia visa ao paciente como um todo, e não focaliza um problema determinado. Dr. Bannerjee, homeopata, oferece uma alegoria para exemplificar as diferentes abordagens terapêuticas:

A força vital e a doença são similares a dois trens de alta velocidade, 'como o TGV francês'. Quando a força da doença sai do controle, ela deve ser parada. Há duas abordagens para parar o trem. A primeira é através de uma colisão completa com o outro trem, que é o método da alopatia; desse modo o trem irá parar rapidamente, mas também haverá grandes danos colaterais. O outro método é enviar um trem por um trilho paralelo que é ainda mais rápido que o primeiro trem, para unir os dois trens e assim diminuir lentamente o trem perigoso. Nessa maneira, a força da doença é extinta sem nenhum dano. Esse segundo método é o método homeopático, efetuando uma alteração na força vital que irá gradualmente extinguindo a força da doença. (Ecks 2014: 2310-2320).

Equilibrar a força vital, tal como propõe a homeopatia, implica o oferecimento de recomendações alimentares durante o processo de diagnóstico. Ecks transcreve um diálogo entre médico e paciente, no qual o primeiro faz apenas quatro perguntas: (1) como você está? (pergunta ampla); (2) como está sua perna? (pergunta específica); (3) algo mais? (pergunta complementar) e, por fim, (4) você sente fome? (pergunta sem relação direta com os sintomas apresentados). Com essas quatro perguntas, o médico rapidamente recomenda a compra de um medicamento. Conquanto esse diálogo possa não ser representativo de todas as consultas locais em homeopatia, ele indica que o foco no sintoma apresentado não é necessariamente relevante, como o seria na dinâmica alopática, e destaca a importância da alimentação.

No quarto e último capítulo, “Psychiatry, Medicating Modern Moods” (“Psiquiatria, medicando humores modernos"), Ecks adentra diretamente o amplo consumo de psicofármacos na região: “Tomar psicofármacos é enquadrado não apenas como um direito, mas como dever de todo cidadão indiano", e complementa a partir da opinião de Dr. Roy (psiquiatra): 
Para ele, psicotrópicos deveriam ser usados como suplementos alimentares, não apenas para tratar doenças, mas também para ajudar pacientes a se tornarem "melhor do que bem". E o alvo deveria ser pensar não apenas no melhoramento individual, mas no aperfeiçoamento de toda a sociedade. (Ecks 2014: 2827).

A popularidade de psicofármacos na região circunscrita pelo autor, onde há elevado uso de medicamentos para humor, contrasta com o aumento das doenças que eles deveriam combater (Ecks 2014: 3438). O uso de tais medicamentos oscila ora no receio do enquadramento enquanto "Iouco", inscrevendo o usuário em uma lógica estigmatizante com relação aos usuários de psicofármacos, e ora na percepção de recuperar um eu anteriormente perdido. É para vencer essa resistência oriunda dos receios que acompanham o uso de psicofármacos que psiquiatras lançam mão da ideia de moner khabar (comida para a mente).

Com isso em vista, entrelaçando em sequência os três sistemas de medicina abordados por Ecks, partese de uma percepção popular e tradicional - uma ideia pré-científica, em termos de Fleck - sobre a importância do sistema digestivo e, por conseguinte, da alimentação; adentra-se o universo da Ayurveda, a qual mantém certo senso de continuidade através de seu fundamento baseado na harmonia do indivíduo com o seu estilo de vida e entorno; perpassa-se a homeopatia, em cuja lógica de formulação de diagnóstico os hábitos alimentares são abordados com a finalidade de criar um vínculo com os pacientes; e, por fim, no universo da psiquiatria, percebe-se uma dinâmica de resistência ao uso de psicofármacos que culmina na utilização da ideia de "comida" para combatê-la. Segundo Ecks, "Para os psiquiatras indianos sintonizados com os conceitos locais de saúde, é apenas um pequeno passo desde reconhecer a centralidade da barriga até descrever psicofármacos como 'comida'" (Ecks 2014: 3456).

Pensar enquanto "comida" altera a percepção acerca dos medicamentos, tornando-os inofensivos, livres do risco de sobredose, e sugerindo um uso contínuo isento do estigma que acoberta aqueles que os utilizam. A partir da visão dos psiquiatras, é a continuidade do tratamento o grande desafio à manutenção da boa saúde. A dificuldade, no entanto, de estabelecer um consenso sobre o que caracteriza a chamada boa saúde, o receio acerca de efeitos adversos associados ao uso prolongado de medicação, e a inclusão - ou explicitação - nessa dinâmica de agentes políticos - via instituição de marcos regulatórios e investimento público em ensino médico - e econômicos propicia certa semelhança ao contexto dos Estados Unidos, conforme trabalhado por Dumit (2012).

"Americanos estão usando drogas" ("Americans are on Drugs", no original), afirma o autor. Esta asserção engloba o aumento do número de tratamentos, além da atuação de indústrias farmacêuticas e suas propagandas - o que, no contexto indiano, é proibido para antidepressivos (Ecks 2014: 2786). A importância da propaganda diz respeito ao aumento da consciência dos pacientes acerca do universo médico no qual foram inscritos. Algo sintomático do processo de biomedicalização (Ecks 2014: 2867) que visa à criação de pacientes/consumidores responsáveis pela manutenção da própria saúde. Conquanto Dumit (2012) percebe a propaganda como uma forma de vinculação direta entre paciente e patologia, Ecks enfatiza que o processo de empoderamento dos pacientes via conscientização era simultaneamente estimulado e desestimulado no ambiente psiquiátrico indiano. Desestimulado justamente em decorrência do risco de reforçar a resistência dos pacientes aos medicamentos a eles prescritos - retornando, assim, à importância de estratégias persuasivas como psicofármacos são "comida para a mente". 
Aumentar a conscientização, em conjunto com o desenvolvimento de novas tecnologias de diagnóstico e de uma ideia de saúde associada ao cuidado e ao risco, estimula tratamentos prolongados. Se para Dumit há uma ideia de "saúde como tratamento" (2012: 217) que implica a noção de cronicidade e, por conseguinte, de tratamentos de longa duração, no contexto elaborado por Ecks a ideia de manutenção da saúde, de atenção continuada, não é problema para os pacientes indianos. Conforme escreve o autor, "O que eles não estão convencidos é de que medicações biomédicas podem e devem ser usadas cronicamente sem causar severos efeitos colaterais" (Ecks 2014: 3595). Aqui, o problema diz respeito ao paradigma da "bala mágica", segundo o qual psicofármacos - e, de modo mais amplo, remédios biomédicos - agiriam especificamente no problema de saúde e evitariam contato com o resto do organismo.

“O modo como médicos indianos tentam convencer seus pacientes a tomarem psicofármacos", escreve Ecks, "é um momento na mudança global de paradigma em direção às "drogas para vida" (Ecks 2014: 3595) - em alusão a Dumit. Diminuir a conscientização dos pacientes sobre os medicamentos e descrevê-los enquanto comida para a mente é, no contexto indiano, uma estratégia para diminuir resistência e conseguir alcançar tratamentos continuados. Drogas para vida enquanto um paradigma médico somente é possível baseado em concepções de saúde como tratamento (de acordo com Dumit) e o anseio pelo melhoramento individual e coletivo ("melhor do que bem", de acordo com Ecks). Ecks e Dumit oferecem, assim, duas diferentes descrições rumo a uma mudança comum de paradigma na utilização de remédios.

Marcos Freire de Andrade Neves é doutorando em Antropologia Social pela Universidade Federal do Rio Grande do Sul (UFRGS) e membro associado (DAAD Fellowship) do grupo Medical Anthropology na Freie Universität Berlin, Alemanha. 


\section{NOTAS}

1 Tradução minha. No original: "What is wrong with taking drugs?" he asked. The pills were simply "nutritional supplements" for the brain. Just as anyone should take vitamins when normal food was not enough, anyone should take food supplements for the brain when nutrition was deficient. The prescribed medicines were moner khabar, and objecting to them was as unwise as objecting to taking vitamin $\mathrm{C}$ while having flu"

2 Todas as traduções foram feitas pelo autor do ensaio bibliográfico. O presente ensaio foi realizado a partir de uma versão digital (e-book), publicada em 2014, pela New York University Press. A versão impressa, por sua vez, foi publicada em 2013. Portanto, atenta-se para a diferença de paginação entre a versão física e a digital: ao invés de páginas, a numeração indicada refere-se à "loc", marcação própria do formato e-book. 
DOMÈNECH, Miquel; TIRADO, Francisco X. "Claves para la lectura te textos simétricos”. In: M. Domènech; F. Tirado. (orgs.). 1998. Sociología simétrica: ensayos sobre ciencia, tecnologia y sociedad. Barcelona: Gedisa.

DUMIT, Joseph. 2012. Drugs for life: How Pharmaceutical Companies Define Our Health. Duke University Press: Durham and London.

ECKS, Stefan. 2014. Eating Drugs: Psychopharmaceutical Pluralism in India (Biopolitics: Medicine, Technoscience, and Health in the 21st Century. NYU Press. (E-book).

FLECK, Ludwik. 2010. Gênese e Desenvolvimento de um Fato Científico. Belo Horizonte: Fabrefactum.

LAW, John; AFDAL, Geir; ASDAL, Kristin; LIN, Wen-yuan, MOSER, Ingunn; SINGLETON, Vicky. 2013. Modes of Syncretism: notes on non-coherence. CRESC Working paper series, 119, Manchester.

MOL, Annemarie. 2002. The body multiple: Ontology in medical practice. Duke University Press.

2008. "Política Ontológica: algumas ideias e várias perguntas". In: J. Nunes; R. Roque (org.) Objectos Impuros. Experiências em estudos sociais da ciência. Porto: Edições Afrontamento.

RICOEUR, Paul. 1983. A Metáfora Viva. Lisboa: Res. 


\section{As percepções sobre psicofármacos na Índia: pensando Eating Drugs, de Ecks}

\section{RESUMO}

O presente ensaio bibliográfico tem seu foco direcionado sobre a etnografia Eating Drugs: Psychopharmaceutical Pluralism in India (Biopolitics: Medicine, Technoscience, and Health in the 21st Century), de Stefan Ecks, no qual o autor empreende uma análise dos usos e percepções sobre o uso de psicofármacos na Índia. A ampla utilização de tais medicamentos é analisado à luz de três sistemas de medicina (Ayurveda, alopatia e homeopatia) e das cooperações e disputas que mobilizam. $O$ trabalho de Ecks é relacionado com outros realizados em contextos distintos, como o de Dumit, nos Estados Unidos.

PALAVRAS-CHAVE: Ayurveda; Biopolítica; Homeopatia; Medicalização; Psicofármacos.

\section{The perceptions on psychopharmaceuticals in India: thinking about Eating Drugs, by Ecks}

\section{ABSTRACT}

This bibliographical essay focuses on the ethnography Eating Drugs: Psychopharmaceutical Pluralism in India (Biopolitics: Medicine, Technoscience, and Health in the 21st Century), by Stefan Ecks, in which the author analyses the uses and perceptions regarding the use of psychopharmaceuticals in India. The widespread use of these drugs is analyzed through three systems of medicine (Ayurveda, allopathy, and homeopathy) and the cooperations and disputes they set in motion. Ecks' work is here correlated to others, carried out in different contexts, such as Dumit's work in the United States.

KEYWORDS: Ayurveda; Biopolitics; Homeopathy; Medicalization; Psychopharmaceuticals. 